\title{
Mini-implants as anchoring devices in the maxila
}

\author{
Emanuelle Morais Pereira ${ }^{1}$, Natalia Souto Outeda Maldonado ${ }^{2}$, William Cunha Brandt ${ }^{2}$, Marcelo Melo Quintela ${ }^{1}$, Danilo Castro Fantini \\ Malavazi $^{1}$ and Caio Vinicius G Roman-Torres ${ }^{1,2 *}$ \\ ${ }^{1}$ Department of Dentistry, Metropolitan University of Santos, SP, Brazil \\ ${ }^{2}$ Department of Dentistry, University Santo Amaro, SP, Brazil
}

\begin{abstract}
Mini-implants have emerged in orthodontics as an anchoring alternative to conventional orthodontic treatments; they have high clinical versatility, facilitating installation in various locations, especially in the inter radicular regions, eliminating problems for the orthodontist such as loss of anchorage. The size of the miniimplants should correspond to the bone available for the insertion site, cylindrical or cylindrical in shape, with a smooth or treated surface. The purpose of this study was to review the literature on the placement of mini-implants as anchoring devices in the maxilla. It is necessary the knowledge of areas with greater thickness of cortical bone to promote stability, as well as the inter-radicular spaces that are safe for the placement of such devices. Its main advantages include its reduced size, easy installation and removal, minimizing the risk of injury to anatomical structures, however it presents as main disadvantage the need for simple surgery that can cause complications if it is not well planned. The safest areas for placement of mini-implants in the maxilla are between the second premolar and first molar. The choice of screw should take into account the mesio-distal space between roots, density, bone depth and mucosal thickness, and factors such as orthodontic movement, direct or indirect anchorage. The anatomy of the chosen site has a considerable individual variation. For the use of the mini-implant, the ideal site should be analyzed by means of computed tomography, panoramic or periapical radiographs.
\end{abstract}

\section{Introduction}

The mini-implants appeared as an alternative to solve problems in the orthodontic clinic, as an anchorage, without the need of patient collaboration, can be used routinely, its possibilities of use are numerous, for ease of installation and removal. The nomenclature varies according to the authors, being also known as mini-implants, mini-bolts or micro-screws. The term mini-implant at the beginning was called a micro-implant, which went out of use because the term "micro" is used when its size is reduced and can only be viewed with microscope magnification features. Other denominations in use are TAD (Temporary Anchorage Device) and MIA (Minimplant Anchorage) [1].

They are characterized in shape, size and design according to the trade mark, they have three distinct parts, which are: head, collar and threadable portion. The head is the part that is exposed, should be small, with polished and rounded surface, and have retentions for placement of orthodontic accessories. The collar corresponds to the smooth surface below the head, may or may not be present in the mini-implant. They present varying lengths, their function is to make the interface between the bone and the external environment, that is, it is covered by the mucosa, which allows variations, to suit the soft tissue thickness of a certain area [2]. They have variations and are manufactured in various sizes and diameters, with lengths of 6.9 and $12 \mathrm{~mm}$ [3] being most commonly used. The threadable portion of the mini-implants have diameters ranging from 1 to $2 \mathrm{~mm}$ and are classified as "self-piercing" when they have a very thin and sharp apex, avoiding the use in most cases of drills for bone perforation.

The "self-threading" has the rounded apex, requiring drilling of the drills in the sites to be placed [4]. Unlike dental implants, the miniimplants are made of titanium alloy, they are smaller in diameter than conventional implants, so it is necessary to use material of higher mechanical resistance than commercially pure titanium, as is the case of the Ti-6AL-4V alloy. This alloy has characteristics inferior to the commercially pure titanium, in relation to the bioactivity, which makes the quality of osseointegration smaller and easier to remove larger $[5,6]$. Among the main advantages, we highlight the reduction in the time of orthodontic treatment, greater comfort to the patient, improving aesthetics, simplification of orthodontic mechanics in complex cases, installation surgery and less invasive removal.

Using a mini-implant with a smaller diameter facilitates insertion between the roots, the risk of fracture is related to the diameter of the mini-implant used, very thin diameters, or whose neck is not resistant at the time of removal. Therefore, it is necessary to use conical pieces with a diameter appropriate to the chosen location. Despite a high success rate, inflammation and infection may occur in the tissues around the mini-implant, contact with the root of the tooth by excessive operator force, and fracture of the mini-implant. Some do not resist rotational forces and complications such as the need for reinstallation in the presence of mobility may arise. Correct control of the surgical technique, application of adequate orthodontic forces, bone density quality at the site of implantation, and control of inflammation in adjacent soft tissues contribute to successful treatment $[1,4,7]$.

\section{Methodology}

This study had as methodology the active search for information in the databases of the MEDLINE, LILACS and BBO, as well as the SciELO

*Correspondence to: Caio Vinicius G Roman-Torres, Rua Professor Eneas de Siqueira Neto, 340, São Paulo, SP, Brazil. Tel: +55 11 21418555; E-mail: cvgrt@hotmail.com

Key words: orthodontic anchorage procedures, dental implantation endosseous, orthodontics

Received: June 15, 2018; Accepted: June 20, 2018; Published: June 26, 2018 
virtual library. It was sought to carry out the bibliographic research on the two central themes of this work: orthodontic mini-implant, dental mini implants, mini-implant anchorage, orthodontic anchorage mini implants. Selected articles were published in English between 1986 and 2016.

\section{Discussion}

The mini-implants appeared in orthodontics as an anchorage alternative, compared to conventional orthodontic treatments, due to their small size, they have high clinical versatility, facilitating the installation in several places, especially in the inter-radicular regions, eliminating problems for the orthodontist such as loss of anchorage. The size of the mini-implants should correspond to the bone available for the cylindrical or tapered cylindrical insertion site with a smooth or treated surface. When positioning the mini-implant it is interesting that there is at least $1 \mathrm{~mm}$ of bone around it to avoid injuries to the teeth and also to facilitate its installation $[3,4]$. The mini-implant is selected according to the location and space available in the same, with the aid of intraoral radiography, a thinner diameter screw is indicated in the maxilla when it is placed between the roots of teeth, a screw of greater length if it is placed in trabecular bone to obtain stability [8].

It is observed a greater thickness of bone in the distal and vestibular region of the second molar, but it is less cortical than in the anterior nasal spine, a greater thickness of cortical bone was found. The apical occlusal orientation of 30 degrees with respect to the bone plane would increase the contact with the cortical bone by up to 1.5 times, compared to the orthogonal orientation of 90 degrees for the placement of mini-implants [9]. In another study, we evaluated that in the maxilla the interradicular region of the posterior teeth, the cortical bone is thicker for installation of the mini-implant in relation to the anterior teeth, these being the places most commonly used for the installation of mini-implants, which are more thicker in adults than in adolescents [10]. The interradicular spaces, either mesial or distal to the first molar, would be the safest place for titanium mini-bolts to be from 1.2 to 1.5 in maximum diameter, with a 6-8 $\mathrm{mm}$ length of cutting thread and conical shape $[11,12]$.

The safe places in the maxilla for placement of mini-implants with adequate space are between the second premolar and the first molar in the vestibular, and between the molars in the palatine [13]. The medial palatine area and the retromolar area are also excellent sites for miniimplant placement, but for Park and Cho 2009 [14], because of limited interradicular spaces, the recommended diameter of a mini-implant is 1,2 to $1.6 \mathrm{~mm}$ for positioning in the alveolar bone, the recommended length is 6-7 mm. The authors Lim et al. [15] and Kim et al. [16] agreed that the best guidelines for installing mini-implants are: apical angulation less than 45 degrees along the axis of the tooth, such as a miniimplant of diameter between 1.4 and $1.8 \mathrm{~mm}$ in length $6 \mathrm{~mm}$ and that the point of contact is near the mucogingival line 2 to $4 \mathrm{~mm}$ from the junction cement enamel. The choice of the mini-implant should take into account the mesio-distal space existing between the roots, the density and thickness of the mucosa, with the aid of panoramic, periapical radiographs and computed tomography.

For greater primary stability, avoid proximity of dental roots, thus installing the micro-screws perpendicularly with an angle of 30 to 40 degrees, in relation to the cortical bone surface. Kim et al; 2006 [17] evaluated that mini-implants may loosen during orthodontic treatment, their stability is related to the quality and quantity of cortical bone, obtained when using computed tomography to investigate (CBT) thickness of the cortical bone in several locations and the location the safest for placement of mini-implant is mesial or distal from the first molar. The micro-bolt should withstand pressure without any mobility, if it is to achieve good primary stability, the micro-bolt should be replaced with a larger one or a new surgical bed should be chosen. This may be due to poor bone density or failure of the micro-bolt installation procedure.

Regarding the installation of the mini-implants can be performed by the technique "transmucosa" the drilling is performed directly with drill or manually, being more indicated in regions of keratinized mucosa and the technique of "surgery with flap". A $5 \mathrm{~mm}$ incision is made, and a mucoperiosteal flap is lifted. The drill is always drilled with a smaller diameter drill than the mini-implant. This technique is more indicated in areas of the alveolar mucosa [18]. The perforation of the buccal cortex of the maxilla is easily performed, because the cortical bone in this region is very thin. The palate region offers greater resistance, especially in the midline, in these cases, a milling cutter of 1.0 $\mathrm{mm}$ in diameter, with a maximum speed of rotation of $300 \mathrm{rpm}$, can be used. After decorticalization, the MPO is installed with a torque of 20 $\mathrm{Ncm}$. It can be considered a success when the orthodontic force could be applied for 6 months without pain or mobility, with a torque of 8 to $10 \mathrm{Ncm}$ [19]. The insertion torque changes according to the thickness of the cortical bone, the thinner the longer the healing time, the thicker the cortical bone the shorter the healing time. The thread design of the mini-implant should be chosen according to the thickness of the cortical bone. The cortical bone has a higher elasticity modulus than the trabecular bone, thus supporting more loads in clinical situations, due to the thickness of the cortical bone, providing greater stability, the use of mini-implants with larger diameters favor the installation, and require of smaller torques for stability, but the use of smaller miniimplants avoids the risks of contact with the root, but may not have a favorable stability, the greater the depth of insertion the better the stability of mini-implants.

Kuroda et al. [20] and Kim et al. [21] agreed that after 12 months of continuous loading the mini-implants were considered successful because they did not present mobility. The time to load and the type of surgery, with flap or transmucosal, do not influence the results for Jason, Sant'ana and Vasconcelos 2005 [3]. Clinical failure of the miniimplant is observed when it presents mobility to vertical or lateral percussion, and gingiva around presenting edemaciada and darker staining, Kim et al; 2010 concluded that vertical angulation for mini-implant placement has a greater result in variability than horizontal angulation. Proximal proximity to the mini-implant combined with maxillary sinus perforation without initial stability was defined as the main risk factor for screw failure.

Contraindications to the use of mini-implants may be classified as absolute or temporary. Temporary contraindications are those related to the difficulty of hygiene at the site of placement of the mini-implant, lack of sufficient space between the roots and in pregnancy due to the occurrence of gingivitis gravidarum. Absolute contraindications are related to patient health status, hematological disorders, type 1 diabetes, patients undergoing radiotherapy, local and systemic disorders. However, the main disadvantage is the need for a simple surgery that can cause complications if it is not well planned. Regarding stability, mini-implants are based on the primary (initial) stability system, not on secondary stability, resulting from osseointegration. Their orthodontic strength is unidirectional, and their retention is mechanical, they can be activated with immediate loading provided they have good stability or late loading [22]. 
The use of mini-implants as temporary anchoring devices was considered an advance in orthodontics, due to its wide versatility of placement, removal, simple surgical technique, small size, variety of lengths that facilitate access in several sites of the maxilla, in addition, they resist orthodontic forces, contribute to an orthodontic treatment without loss of anchorage, being considered one of the great factors of failure in orthodontic treatments, the mini-implants can promote maximum anchorage, and this anchorage can be controlled minimizing unwanted effects, and does not require patient collaboration.

We conclude that the use of mini-implants in orthodontics promotes a considerable improvement in skeletal anchorage, reducing treatment time and a favorable prognosis, improving the control of orthodontic appliance use.

\section{References}

1. Costa A, Raffainl M, Melsen B (1998) Miniscrews as orthodontic anchorage: a preliminary report. Int J Adult Orthodon Orthognath Surg 13: 201-209. [Crossref]

2. Nienkemper M, Handschel J, Drescher D (2014) Systematic review of mini-implant displacement under orthodontic loading. Int J Oral Sci. 6:1-6.

3. Cousley RR (2010) A clinical strategy for maxillary molar intrusion using orthodontic mini-implants and a customized palatal arch. J Orthod. 37:202-208.

4. Meursinge Reynders R, Ronchi L, Ladu L, Di Girolamo N, de Lange J, et al. (2016) Barriers and facilitators to the implementation of orthodontic mini implants in clinical practice: a systematic review. Syst Rev. 5:163.

5. Abbassy MA, Bakry AS, Zawawi KH, et al. (2018) Long-term durability of orthodontic mini-implants. Odontology 106: 208-214. [Crossref]

6. Nienkemper M, Santel N, Hönscheid R, Drescher D (2016) Orthodontic mini-implant stability at different insertion depths : Sensitivity of three stability measurement methods. J Orofac Orthop 77: 296-303. [Crossref]

7. Yao CC, Chang HH, Chang JZ, Lai HH, Lu SC, et al. (2015) Revisiting the stability of mini-implants used for orthodontic anchorage. J Formos Med Assoc 114: 1122-1128. [Crossref]

8. Dalla Rosa F, Burmann PF, Ruschel HC, Vargas IA, Kramer PF (2016) Evaluation of fracture torque resistance of orthodontic mini-implants. Acta Odontol Latinoam 29: 248-254. [Crossref]
9. Deguchi T, Nasu M, Murakami K, Yabuuchi T, Kamioka H, Takano-Yamamoto T (2006) Quantitative evaluation of cortical bone thickness with computed tomographic sacanning for orthodonticimplantes. Am J Orthod Dentofacial Orthop. 129:721 e 7-12.

10. Farnsworth D, Rossouw PE, Ceen RF, Buschang PH (2011) Cortical bone thickness at common miniscrew implant placement sites. Am J Orthod Dentofacial Orthop 139 495-503. [Crossref]

11. Poggio PM, Incorvati C, Velo S, Carano A (2006) "Safe zones": a guide for miniscrew positioning in the maxillary and mandibular arch. Angle Orthod 76: 191-197. [Crossref]

12. Baumgaertel S, Hans MG (2009) Buccal cortical bone thickness for mini-implant placement. Am J Orthod Dentofacial Orthop 136: 230-235. [Crossref]

13. Park HS, Lee YJ, Jeong SH, Kwon TG (2008) Density of the alveolar and basal bones of the maxilla and the mandible. Am J Orthod Dentofacial Orthop 133: 30-37. [Crossref]

14. Park J, Cho HJ (2009) Three-dimensional evaluation of inter radicular spaces and cortical bone thickness for the placement and initial stability of micro implants in adults. Am.J. Orthod. Dentofacial Orthop. 136:314 e 1-2; discussion 314-5.

15. Lim JE, Lim WH, Chun YS (2008) Quantitative evaluation of cortical bone thickness and root proximity at maxillary interradicular sites for orthodontic mini-implant placement. Clin Anat. 21:486-91.

16. Kim SH, Yoon HG, Choi YS, Hwang EH, Kook YA, Nelson G (2009) Evaluation of interdental space of the maxillary posterior area for orthodontic mini-implants with cone-beam computed tomography. Am J Orthod Dentofacial Orthop. 135:635-41.

17. Kim HJ, Yun HS, Park HD, Kim DH, Park YC (2006) Soft-tissue and cortical-bone thickness at orthodontic implant sites. Am J Orthod Dentofacial Orthop 130: 177-182. [Crossref]

18. Park HS, Bae SM, Kyung HM, Sung JH (2004) Silmultaneous incisor retraction and distal molar movement with micro-implant anchorage.World J Orthod. 5:164-167.

19. Motoyoshi M, Yoshida T, Ono A, Shimizu N (2007) Effect of cortical bone thickness and implant placement torque on stability of orthodontic mini-implants. Int J Oral Maxillofac Implants 22: 779-784. [Crossref]

20. Kuroda S, Yamada K, Deguchi, Hashimoto T, Kyung HM, Takano-Yamamoto T (2007) Root proximity is a major factor for screw failure in orthodontic anchorage. $\mathrm{Am} \mathrm{J}$ Orthod Dentofacial Orthop 131(4Suppl):S68-73.

21. Kim SH, Kang SM, Choi YS, Kook YA, Chung KR, Huang JC (2010) Cone-beam computed tomography evaluation of mini-implants after placement: Is root proximity a major risk factor for failure? Am J Orthod Dentofacial Orthop. 138:264-76.

22. Assad-Loss TF, Kitahara-Céia FMF, Silveira GS, Elias CN, Mucha JN (2017) Fracture strength of orthodontic mini-implants. Dental Press J Orthod 22: 47-54. [Crossref]

Copyright: $₫ 2018$ Pereira EM. This is an open-access article distributed under the terms of the Creative Commons Attribution License, which permits unrestricted use, distribution, and reproduction in any medium, provided the original author and source are credited. 